\title{
OPEN Laser-scribed graphene nanofiber decorated with oil palm lignin capped silver nanoparticles: a green biosensor
}

\author{
Melvin Jia Yong Tai ${ }^{1,2}$, Veeradasan Perumal ${ }^{1,2 \bowtie}$, Subash C. B. Gopinath ${ }^{3,4 凶}$, \\ Pandian Bothi Raja ${ }^{5}$, Mohamad Nasir Mohamad Ibrahim ${ }^{5}$, Iffah Najihah Jantan ${ }^{5}$, \\ Nur Syahirah Husna Suhaimi ${ }^{5} \&$ Wei-Wen Liu ${ }^{3}$
}

Tuberculosis (TB), caused by Mycobacterium tuberculosis (M. tuberculosis), requires a high level of attention and is one of the most infectious diseases in the air. Present methods of diagnosing TB remain ineffective owing to their low sensitivity and time consumption. In this study, we produced a green graphene nanofiber laser biosensor (LSG-NF) decorated with oil palm lignin-based synthetic silver nanoparticles (AgNPs). The resulting composite morphology was observed by field-emission scanning electron microscopy and transmission electron microscopy, which revealed the effective adaptation of the AgNPs to the LSG-NF surface. The successful attachment of AgNPs and LSG-NFs was also evident from X-ray diffraction and Raman spectroscopy studies. In order to verify the sensing efficiency, a selective DNA sample captured on AgNPs was investigated for specific binding with M.tb target DNA through selective hybridisation and mismatch analysis. Electrochemical impedance studies further confirmed sensitive detection of up to $1 \mathrm{fM}$, where a detection limit of $10^{-15} \mathrm{M}$ was obtained by estimating the signal-to-noise ratio $(S / N=3: 1)$ as $3 \sigma$. Successful DNA immobilisation and hybridisation was confirmed by the detection of phosphorus and nitrogen peaks based on $X$-ray photoelectron spectroscopy and Fourier-transform infrared spectroscopy. The stability and repeatability of the analysis were high. This approach provides an affordable potential sensing system for the determination of $M$. tuberculosis biomarker and thus provides a new direction in medical diagnosis.

Tuberculosis (TB) is considered on par with HIV/AIDS as a major disease ${ }^{1}$, affecting billions of people and causing millions of deaths each year. Mycobacterium tuberculosis (M. tuberculosis) is the key pathogen responsible for TB. The World Health Organization (WHO) adopted the 'End TB Strategy' to eliminate this disease by 2035 and obtained a positive response from most countries ${ }^{2}$. These countries have introduced a variety of new approaches to reduce TB epidemics. However, the current techniques for diagnosing this disease suffer from low sensitivity and require prolonged times to obtain correct results ${ }^{3}$. Rapid, cost-effective, and high-sensitivity biosensors are therefore required for the effective detection of TB in clinical samples ${ }^{4}$.

Carbon-based nanomaterials such as graphene, fullerene, and carbon nanotubes (CNTs) have played an important role in various applications owing to their specific properties ${ }^{5}$. The design of these carbon nanomaterials has a direct impact on their electron transfer rate and therefore affects their analytical efficiency ${ }^{6}$ in areas such as electrochemical sensing ${ }^{7}$. Interestingly, graphene has received much attention in electrochemical research. These carbon-based materials possess many beneficial properties, such as high thermal and electrical conductivity. Previous reports indicate that graphene displays excellent electrochemical properties when it adopts a porous $3 \mathrm{D}$ network pattern, enabling the mass transfer of charged electrons. While graphene provides

\footnotetext{
${ }^{1}$ Centre of Innovative Nanostructures \& Nanodevices (COINN), Universiti Teknologi PETRONAS (UTP), Seri Iskandar, Perak, Malaysia. ${ }^{2}$ Mechanical Engineering Department, Universiti Teknologi PETRONAS (UTP), Seri Iskandar, Perak, Malaysia. ${ }^{3}$ Institute of Nano Electronic Engineering, Universiti Malaysia Perlis (UniMAP), Kangar, Perlis, Malaysia. ${ }^{4}$ Faculty of Chemical Engineering Technology, Universiti Malaysia Perlis (UniMAP), Arau, Perlis, Malaysia. ${ }^{5}$ School of Chemical Sciences, Universiti Sains Malaysia, Gelugor, Penang, Malaysia. ${ }^{\square}$ email: veeradasan.perumal@utp.edu.my; subash@unimap.edu.my
} 
outstanding functionality, graphene manufacturing processes are costly and complicated ${ }^{5}$. Therefore, the industrial production of graphene devices in a cost-effective manner is mandatory.

Previous research has reported a simpler and efficient method of graphene synthesis by penetrating a commercial polyimide (PI) film with a $\mathrm{CO}_{2}$ laser in an ambient graphene-scribing laser (LSG) setting ${ }^{8}$. Surprisingly, the recorded process generated a porous $3 \mathrm{D}$ network pattern, affording highly defective graphene, but offering high electrical conductivity and stability, resulting in excellent electrochemical performance for sensing applications. A novel graphene product called laser-induced graphene fibre (LIGF) was also obtained by monitoring the amount of radiation energy entering the polyimide film, where the quality of graphitization was evaluated by using a LIGF-formed microsupercapacitor ${ }^{9}$. In addition, several studies have revealed the efficacy of LSG in transporting electrons in biochemical reactions, thus serving as a perfect interface platform for biomolecules ${ }^{5-7,10}$.

The use of graphene as a standalone electrode for sensing devices has been documented, but the electrochemical efficiency of the developed electrodes requires further enhancement ${ }^{11-13}$. The introduction of nanoparticles into such bioelectrodes has therefore become a preferred technique for enhancing the selectivity and sensitivity ${ }^{14}$. As far as economy and conductivity are concerned, copper nanoparticles (CuNPs) are the preferred choice for sensor applications, while custom synthesis conditions and easy oxidation in the absence of capping agents make CuNPs an undesirable material ${ }^{15}$. The ultrafast/facile biosynthesis of gold nanoparticles (AuNPs) and silver nanoparticles (AgNPs) using bio-materials has propelled their applications in sundry research fields within a short period. Due to the lower cost of synthesising AgNPs relative to that of AuNPs and the same excellent results achieved with both species in the biosensor research field, AgNPs were selected as the top material choice for the present study ${ }^{16-18}$.

Chemical reduction of silver metal into AgNPs is a traditional approach in which various synthetic reducing agents including $\mathrm{NaBH}_{4}$, sodium citrate, hydrazine, etc., have been successfully employed ${ }^{19,20}$. Although these chemicals produce stable AgNPs with the desired particle size, their toxicity has a huge impact on the environment, thus forcing scientists to find green/compatible replacements. Oil palm-derived lignin has many phenolic hydroxyl groups on its molecular skeleton; thus, lignin has been established as an ideal, green, and potential reducing agent for AgNP synthesis. Our group recently developed an eco-friendly and cost-effective method for synthesising silver nanoparticles (AgNPs) from palm oil lignin. Owing to its biocompatibility, high conductivity, and low toxicity, an increase in the analytical performance of the LSG bioelectrode was predicted ${ }^{16}$.

The current work presents a method of manufacturing graphene nanofibers by laser-scribing after modification with oil palm lignin-capped AgNPs to allow direct bonding with a single strand of DNA for the production of a TB bioelectrode. The bioelectrode is characterised morphologically and structurally, and its selectivity and sensitivity are investigated. Decorated laser graphene nanofiber (LSG-NF-AgNP) bioelectrodes provide an avenue for the generation of versatile and high-sensitivity sensing platforms.

\section{Results and discussion}

The bioelectrode was fabricated by penetrating a PI film with a $\mathrm{CO}_{2}$ laser to produce a laser-scribed graphene nanofiber (LSG-NF), where PI works as the substrate on the device. AgNPs were green-synthesised from oil palm lignin (the formation and characterisation are presented in the "Supplementary Information"), which was then added to the LSG-NF by the drop-cast method. The modification creates defects on the LSG-NF and facilitates the accommodation of DNA, increasing the sensitivity of the bioelectrode (Fig. 1a). The surface was characterised by morphological, structural, and chemical analyses to confirm the performance of the biosensor.

Field-emission scanning electron microscopy (FESEM). The overall morphology of the LSG-NFs decorated with silver nanoparticles (AgNPs) was characterised by FESEM. Figure 1b,c display the morphological images of LSG-NF obtained before and after introducing the AgNPs using the drop-cast method. Figure 1b shows the structure of the fabricated LSG-NF, which forms a smooth hair-like structure with diameters ranging from 155 to $290 \mathrm{~nm}$. Figure 1c,d show the morphology of the LSG-NF decorated with shiny particles, whereby AgNPs attached on the entire surface grew at a high density to generate a coral-like structure. Clear agglomeration of the AgNPs was observed, indicating homogeneity. Careful observation of the magnified image (Fig. 1d) revealed that the entire surface of the LSG-NF became coarse and the average diameter increased to $300 \mathrm{~nm}$ upon accommodation of the AgNPs. This roughened surface affords a higher surface area, providing vacancy defects, which can facilitate the chemisorption of biomolecules on the substrate and permit more facile stacking of DNA during the immobilisation and hybridisation processes ${ }^{21,22}$.

Transmission electron microscopy (TEM). The surface morphology of the LSG-NFs and AgNPs was further characterised by TEM. Figure 2 shows the high- and low-magnification TEM images of the LSG-NF fabricated before and after agglomeration of the AgNPs. The low-magnification TEM images of LSG-NF in Fig. 2a reveal the fibre structure of LSG without a hollow core, indicating that the fabricated specimen is not a carbon nanotube. The observation at higher magnification (Fig. 2b) reveals that the diameter of LSG is $\sim 290 \mathrm{~nm}$, which is similar to the FESEM results. After introduction of the AgNPs onto LSG, a few black spots were found to be deposited on the graphene layer (Fig. 2c). The higher magnification TEM image in Fig. 2d shows spherical AgNPs as black spots with an average diameter of $19 \mathrm{~nm}$, localized on the edge of LSG. Thus, AgNPs were observed to be randomly attached to the LSG-NF and increased the overall diameter of the specimen ${ }^{21}$.

X-ray diffraction (XRD). X-ray diffraction was used to inspect the crystal structure, plane scale, and orientation of the fabricated AgNP-decorated LSG-NFs. The XRD spectra of the AgNPs decorated with LSG-NF are compared with those of plain LSG-NF in Fig. 3a. The XRD patterns presented peaks at $25.96^{\circ}(002)$ and $46.26^{\circ}(101)$, attributed to graphene (black spectrum). Remarkably, three extra diffraction peaks appeared in the 


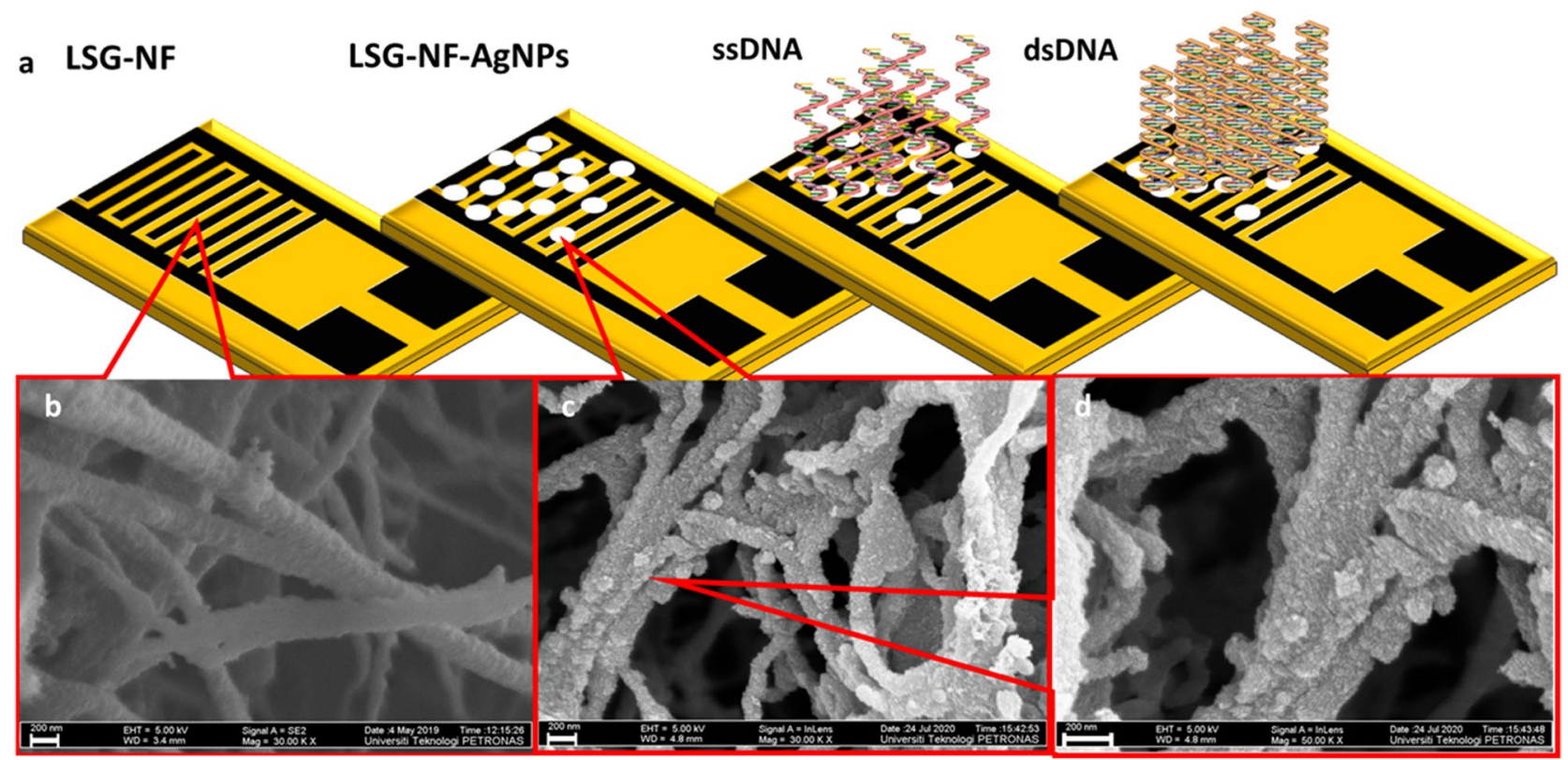

Figure 1. (a) Schematic diagram illustrating the process involved in the fabrication of LSG-NF-AgNPs DNA bioelectrode. (b) Low-magnification FESEM image showing smooth hair-like structure of synthesized LSG-NF: low- (c) and (d)- high magnification images of AgNP-decorated LSG-NF. Images show coral-like structure decorated with shiny particles.

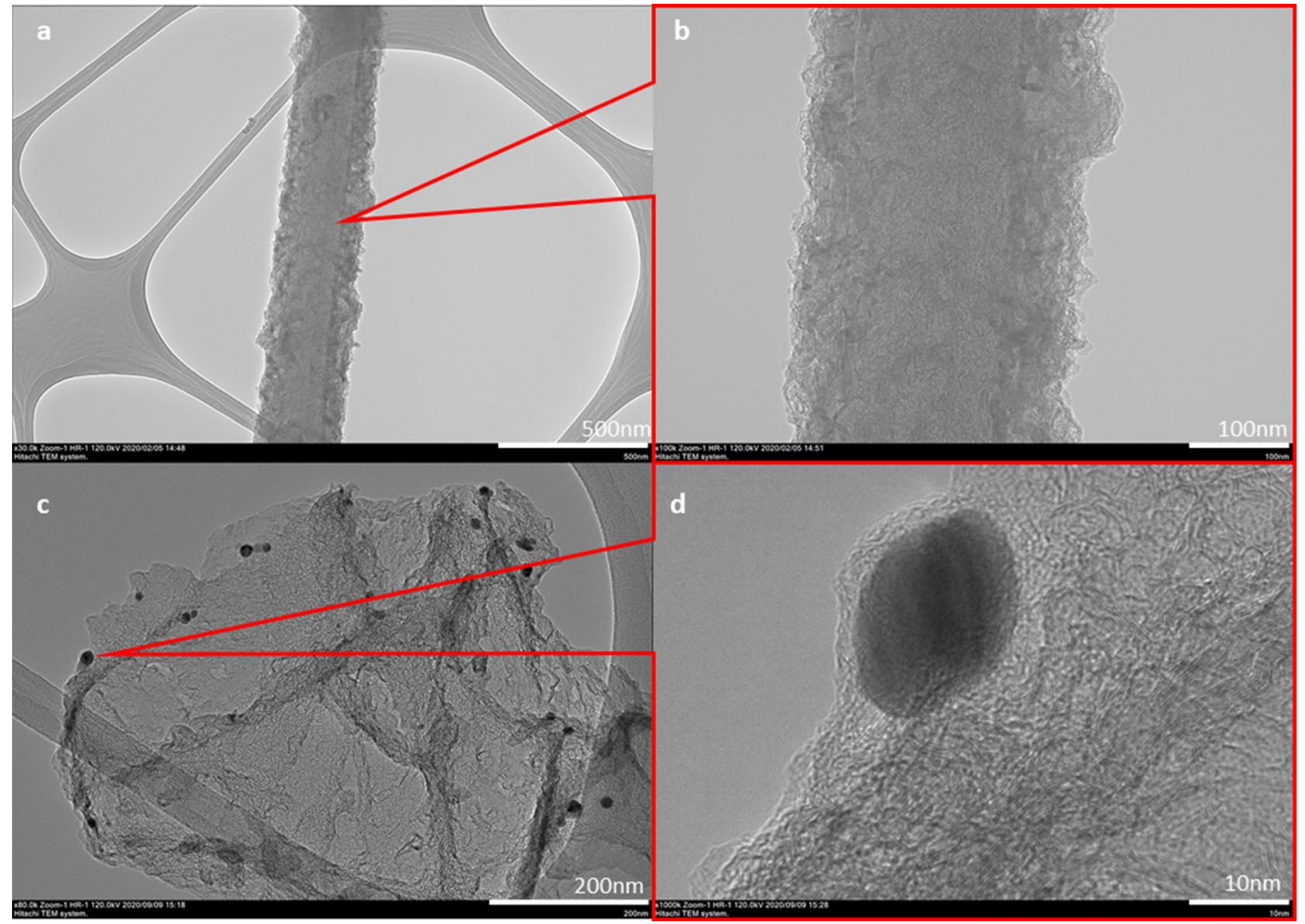

Figure 2. Typical TEM images of (a) LSG-NF and (b) LSG-NF-AgNPs, (c, d) shows high magnification TEM images of LSG-NF and LSG-NF-AgNPs, respectively. 

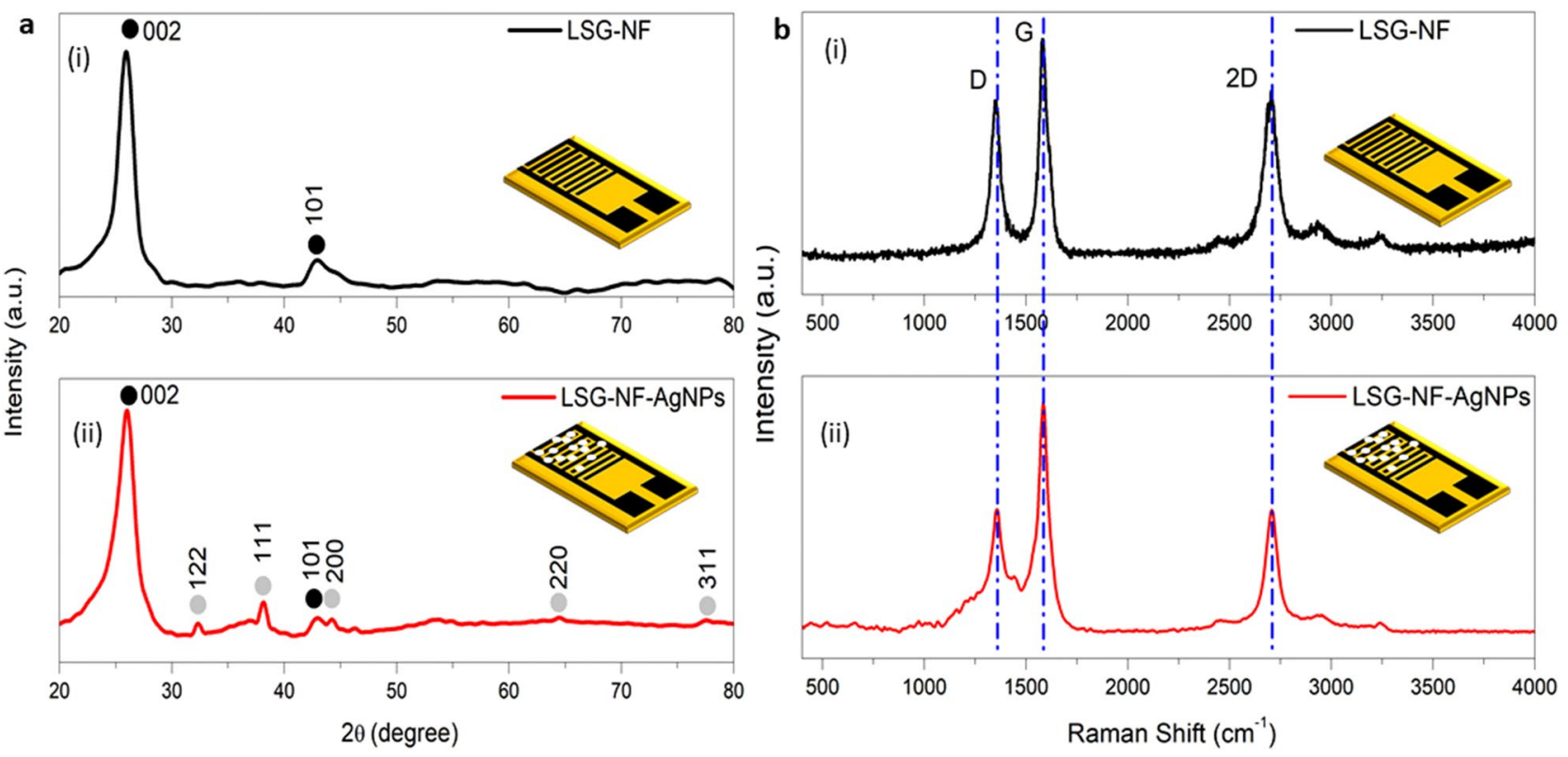

Figure 3. (a) X-ray diffraction and (b) Raman spectra of LSG-NF, (i) before and (ii) after adding AgNPs.

profile of the LSG-NF-AgNPs (red spectrum) relative to that of pure LSG-NF. The diffraction peaks at $33.03^{\circ}$, $38.75^{\circ}, 44.27^{\circ}, 64.57^{\circ}$, and $77.44^{\circ}$ were assigned to the diffraction lines of the (122), (111), (200), (220), and (311) planes, respectively, which suggests that the AgNPs have a face-centred cubic structure with a crystalline morphology ${ }^{23-26}$. No diffraction peak due to lignin was observed in the XRD pattern, as lignin is amorphous in nature and lacks an ordered structure ${ }^{27,28}$. The sharp and narrow diffraction peaks indicate that LSG-NF is highly crystalline.

Raman spectroscopy. The Raman spectra of pure LSG-NF and AgNP-decorated LSG-NF are displayed in Fig. 3b. The Raman spectra show typical sharp D, G, and 2D bands at $1358 \mathrm{~cm}^{-1}, 1584 \mathrm{~cm}^{-1}$, and $2708 \mathrm{~cm}^{-1}$, respectively, indicative of the hexagonal graphene lattice. The $\mathrm{D}$ band is known as a disorder or defect band. In both spectra, the D band was relatively intense, indicating imperfect arrangement of the LSG-NF and AgNPs, which allows accommodation of the single-stranded DNA on these substrates and enhances the sensitivity of the biosensor. The intensity ratio of the D and G peaks of LSG-NF (0.75) was slightly reduced to 0.52 upon addition of the AgNPs, indicating that the presence of the AgNPs successfully decreased the defects in LSG-NF ${ }^{29-31}$. The $I_{2 D} / I_{G}$ ratio of LSG-NF was calculated to be 0.79 , indicating multilayered graphene. For the LSG-NF-AgNPs, the intensity of all three bands was compared to that of the pure LSG-NF, which showed scattering at a lower Raman shift, possibly due to the chemical bonds formed between the lignin molecules and silver, amino nitrogens, and carboxylate groups.

X-ray photoelectron spectroscopy (XPS). XPS was used to study the elemental composition and chemical state of the elements present on the outermost layer of the AgNP-decorated LSG-NFs and the degree of transformation on the surface upon functionalization for the detection of $M$. tuberculosis. In the full XPS survey scans (Fig. 4a) of all three samples analysed, photoelectron peaks of carbon, oxygen, silver, sulphur, phosphorus, and nitrogen were observed. The binding energy peaks at $284 \mathrm{eV}$ and $532 \mathrm{eV}$ correspond to $\mathrm{C} 1 \mathrm{~s}$ and $\mathrm{O} 1 \mathrm{~s}$, respectively. Ag3d peaks were also observed at 368 and $374 \mathrm{eV}$. Upon functionalization, the intensity of the $\mathrm{C} 1 \mathrm{~s}$ and O1s peaks increased slightly, whereas that of the Ag3d state decreased. This phenomenon indicates the effective immobilisation and hybridisation of the developed bio-electrode. S2p peaks were observed at 163 and $168 \mathrm{eV}$, attributed to the thiol-terminated probe DNA. In addition, two elements (P2p and N1s) were observed after immobilisation and hybridisation. This supports the FTIR results, where phosphorus and nitrogen functional groups are bound on the bio-electrode. It was also found that the binding energy of both the immobilisation and hybridisation peaks changed slightly due to the formation of a bond between the probe DNA and target DNA on the bioelectrode surface. Therefore, the capability of immobilizing and hybridising the bioelectrode was confirmed from the XPS results.

Confirmation of immobilization and hybridization through Fourier-transform infrared (FTIR) spectroscopy. FTIR analysis provides details on the chemical compounds present on the outermost layer of the AgNPs decorated with LSG-NF after immobilisation and hybridisation of $M$. tuberculosis DNA sequences based on their vibrational signature. The FTIR spectra of the bare LSG-NF, immobilised LSG-NF (probe)-decorated AgNPs, and hybridised LSG-NF (duplex)-decorated AgNPs are shown in Fig. 4b. The chemical bonds in the functional groups of molecules attenuate the transmission of infrared radiation through stretching and bending vibrations. Prior research has generally confirmed that the $700-1800 \mathrm{~cm}^{-1}$ range of absorption or transmittance is the fingerprint area for nucleic acids ${ }^{32,33}$. Figure $4 \mathrm{~b}$ presents a comparison of the FTIR spectra of 

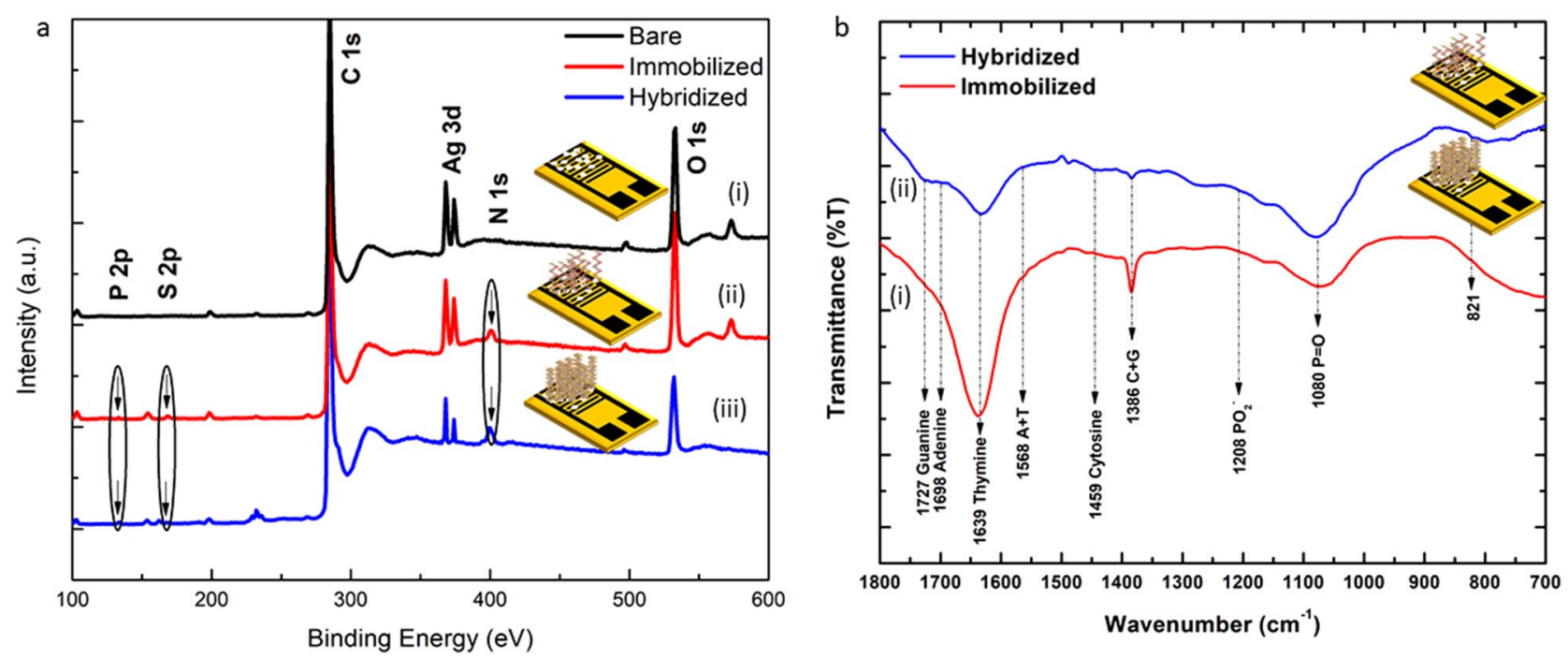

Figure 4. (a) Survey scan of XPS core level spectra for (i) LSG-NF-AgNPs, (ii) immobilized (LSG-NF-AgNPs/ ssDNA) and hybridized (LSG-NF-AgNPs/dsDNA), (b) FTIR spectra of LSG-NF-AgNPs bioelectrode upon (i) immobilization and (ii) hybridization of DNA sequences from Mycobacterium tuberculosis. Transmittance region: $700-1800 \mathrm{~cm}^{-1}$.

the immobilised (ssDNA) and hybridised (dsDNA) surfaces, displaying approximately identical transmittance peaks with minor variations. The presence of deoxyribose phosphate in both the probe and duplex spectra was indicated by the peak at $821 \mathrm{~cm}^{-1}$ and an insignificant vibrational band. The peak moved from 900 to $1250 \mathrm{~cm}^{-1}$ upon hybridization. Additional peaks were observed in the $900-1250 \mathrm{~cm}^{-1}$ range, suggesting enhanced symmetric and asymmetric vibrations of the $\mathrm{PO}_{4}{ }^{3-}$ group of the probe and target DNA derived from the DNA phosphodiester deoxyribose backbone. The FTIR spectra of both ssDNA and the duplex display absorption peaks at 1080 and $1208 \mathrm{~cm}^{-1}$, indicating the presence of DNA phosphate classes ${ }^{34}$. The efficacy of immobilisation and hybridisation of the AgNPs on the decorated LSG-NF bioelectrode was confirmed by the peaks at 1460, 1639, 1698 , and $1727 \mathrm{~cm}^{-1}$, corresponding to the respective functional groups, that is cytosine, thymine, adenine, and guanine $^{35}$. In brief, the effectiveness of functionalization on the bioelectrode was corroborated by the FTIR data.

Bio-sensing analyses on AgNPs-decorated laser-scribed graphene nanofiber. Electrochemical impedance spectroscopy (EIS) was used to examine the biosensing activity of the AgNPs decorated with LSGNF. The data acquired over the frequency range of $100 \mathrm{~Hz}-1 \mathrm{MHz}$ with a $1 \times \mathrm{PBS}$ solution $(\mathrm{pH} 7.4)$ were used to construct Nyquist plots. Nyquist plots generally show a semicircle at higher frequencies, which denotes the interfacial load transfer resistance $\left(R_{c t}\right)$, which correlates to carrier transfer from the bioelectrode to the PBS solution, followed by a linear component at lower frequencies that contributes to the diffusion-limited phase, as shown in Fig. $5 \mathrm{a}$. The acquired Nyquist plot can be expressed by the Randles equivalent circuit (inset), where $R_{s}$ and $R_{c t}$ represent the bulk solution resistance and charge transfer resistance, respectively. $Z_{w}$ represents the Warburg impedance, and CPE is the constant phase element. As seen in Fig. 5a, bare LSG-NF exhibited a higher $R_{c t}(\sim 17 \mathrm{~K})$ owing to the presence of imperfections in the LSG-NF. After decoration with the AgNPs, the $R_{c t}$ was significantly reduced $(\sim 6 \mathrm{~K})$, indicating that the bare system undergoes excellent electron transfer to the PBS solution owing to its wide surface area ${ }^{29}$. After the immobilisation phase, the $R_{c t}$ increased to $\sim 8.5 \mathrm{~K}$, leading to the adsorption and immobilisation of the ssDNA by the Ag-SH bond on the surface, slowing the diffusion of the electrolyte to the system surface. The improvement in $R_{c t}$ indicates effective immobilisation of the DNA probe and reduced electrostatic repulsion, attributed to the presence of the negatively charged phosphate skeletons in $\mathrm{DNA}^{21}$. The $R_{c t}$ was further increased to $\sim 10.5 \mathrm{k} \Omega$ during the hybridisation process. The increased $R_{c t}$ value is due to the additional phosphate skeleton from the target DNA, inducing more electrostatic repulsion on the electrosurface. This proves the effective hybridisation of the target DNA on the immobilised device, forming a double-stranded DNA duplex on the device surface. The Nyquist plot was constructed for the immobilised electrode exposed to different concentrations of the target DNA ( $1 \mathrm{fM}-1 \mathrm{nM}$ ) (Fig. $5 \mathrm{~b}$ ). The $R_{c t}$ value increased with increasing concentrations of the target DNA upon hybridisation. This phenomenon is due to the increasing electrostatic repulsion between the negative ion and the negatively charged phosphate backbone found in the probe DNA, which led to the significant change in $R_{c t}{ }^{10}$. To investigate the sensitivity of the AgNP-decorated LSG-NF biosensor, a linear correlation with the difference in the $R_{c t}$ was plotted according to the equation:

$$
\Delta R_{c t}=R_{c} t_{\text {hybridization }}-\text { Rct }_{\text {immobilization }}
$$

corresponding to the logarithm of the complementary DNA concentrations (Fig. 5c). As shown in Fig. $5 \mathrm{c}, \Delta R_{c t}$ increased linearly as the complementary DNA concentration increased. The differences in the values $\left(\Delta R_{c t}\right)$ between the $R_{c t}$ of the immobilized and hybridized species were found to fit the natural logarithm of the target DNA concentrations according to the linear relation: $\Delta R_{c t}=0.812 \times 10^{3} \mathrm{x}+3.673 \times 10^{4}$ with a regression coefficient $\left(R^{2}\right)$ of 0.9896 . 

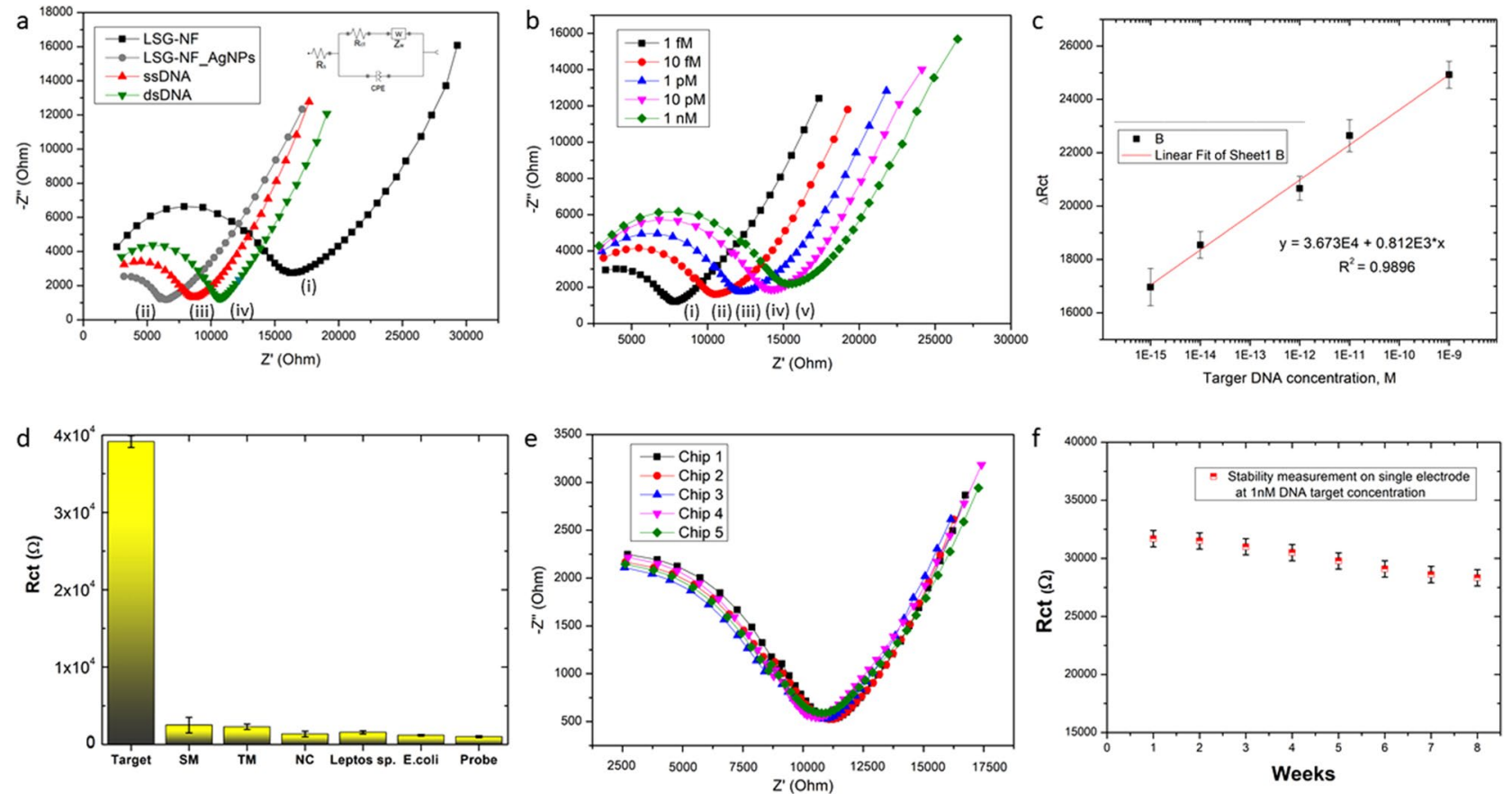

Figure 5. (a) Impedimetric curve of (i) LSG-NF, (ii) LSG-NF-AgNP, (ii) immobilized LSG-NF-AgNPs (probe), and (iii) hybridized LSG-NF-AgNPs (duplex) bioelectrode; the inset shows the Randles equivalent circuit, where the parameters $R_{s}, R_{c t}, Z_{w}$, and CPE represent the bulk solution resistance, charge transfer resistance, Warburg impedance, and constant phase element respectively. (b) Impedance spectra of LSG-NF-AgNPs hybridized with different concentrations of complementary target DNA (i-v) $1 \mathrm{fM}$ to $1 \mathrm{nM}$, (c) illustrates the linear regression curve at different concentrations of target DNA with the linear equation: $\Delta R_{c t}=0.812 \times 10^{3} \mathrm{x}+3.673 \times 10^{4}$, $\left(R^{2}=0.9896\right)$, (d) bar chart showing specificity of the LSG-NF-AgNP bioelectrode against mismatching and cross hybrids, (e) reproducibility curve of 5 parallel bioelectrodes fabricated under similar processing conditions, (f) stability of LSG-NF-AgNP biosensors.

Bio-sensing analyses on Ag-decorated laser-scribed graphene nanofiber. We further investigated the analytical performance of the AgNP-decorated LSG-NF bioelectrode, as displayed in Fig. $5 \mathrm{~d}-\mathrm{f}$. The electrode provided favourable linearity, with detection and quantification limits of $\sim 10^{-15} \mathrm{M}$ and $\sim 10^{-13} \mathrm{M}$, respectively. The results were determined by estimating the signal-to-noise ratio as $3 \sigma$ and $10 \sigma$, respectively ${ }^{36}$. The $R_{c t}$ for the target DNA $(1 \mathrm{nM})$ was $\sim 39 \mathrm{k} \Omega$, which is approximately 16 times greater than the $R_{c t}$ of single-base mismatched DNA $(\sim 2.5 \mathrm{k} \Omega)$ at $1 \mathrm{nM}$, signifying that the biosensor has brilliant sequence sensitivity towards even a singlebase mismatch. Thus, the obtained sensitivity is among the best outcomes reported ${ }^{12}$. Likewise, there was no complementation between the probe and triple mismatched DNA. The cross-reactivity with Escherichia coli and Leptospira species/serovars is shown in Fig. $5 \mathrm{~d}$. The $R_{c t}$ values were not significantly affected by non-target substances, which suggests that non-target substances do not induce any specific interference. The repeatability of the analysis employing the AgNP-decorated LSG-NF bio-electrode was studied by comparing five samples from the same batch prepared under similar conditions. The repeatability curve for the developed bioelectrode is shown in Fig. 5e, which shows the relative standard deviation (RSD) obtained from five parallel measurements for samples prepared under the same processing conditions (3\%). Finally, the stability of the developed bioelectrode was investigated by conducting a shelf-life study over a period of 8 weeks with storage at $4{ }^{\circ} \mathrm{C}$; measurements were performed at a regular interval of 1 week. The results reveal that the developed biosensor is relatively stable and suffered from only 10\% degradation of its activity throughout the 8 weeks of measurement (Fig. 5f).

\section{Conclusions}

The presence of AgNPs in the LSG-NF bioelectrode created a novel biosensitivity-enhanced framework for the identification of Mycobacterium tuberculosis. The morphology of the unit was initially characterised by FESEM and TEM, which revealed that the prepared AgNPs were successfully accommodated on LSG-NF. Furthermore, LSG-NF was characterized by XRD and Raman spectroscopy prior to DNA immobilisation and hybridisation analysis. Added proof was provided by studying the surface chemical groups on the hybridised DNA surface. These findings clearly demonstrate the stability, repeatability, and selectivity up to the femtomolar level. Hence, this electrode could potentially contribute to the medical industry.

\section{Methods}

Materials and reagents. A 5 Mil polyimide (PI) film was provided by Dye Solar Cell Laboratory, Centre of Innovative Nanomaterials and Nanodevices (COINN), Universiti Teknologi PETRONAS (UTP). (3-Aminopropyl)trimethoxysilane (APTES) ( $\geq 98 \%$; Sigma Aldrich, St. Louis, Missouri, USA), diluted in $2 \%$ ethanol 
as a solvent $\left(\mathrm{CH}_{3} \mathrm{CH}_{2} \mathrm{OH}\right.$; 95\%; HmbG Chemicals, Germany) and silver nanoparticles were prepared using oil palm lignin and aqueous silver nitrate solutions (2.5\% w/v $\mathrm{AgNO}_{3}$ in $\mathrm{H}_{2} \mathrm{O}$, Sigma Aldrich; St. Louis, Missouri, USA) and were used for surface modification of the interdigitated electrode (IDE). Ethanolamine $\left(\mathrm{C}_{2} \mathrm{H}_{7} \mathrm{NO}\right.$; Sigma Aldrich; St. Louis, MO, USA) was used as the blocking agent. Phosphate-buffered saline (PBS) (1X; First BASE Biochemicals, Singapore) was used to maintain the optimal $\mathrm{pH}$ and improve the binding events during the impedance measurements. A 21-mer oligonucleotide with the following sequence was used in this study:

thiolated probe DNA (p-DNA): 5-CCG TGA TTT TCC TAA CTA AGG/3ThioMC3-D-3'; complementary target DNA (t-DNA):3'-GGC ACT AAA AGG ATT GAT TCC-5'; non-complementary target DNA (nc-DNA): 3'-G ATT CTG CCG CTT GGC TGC CAA-5'; one-base mismatched target DNA (m-DNA): 3'-GGC ACT AAA ATG ATT GAT TCC-5'; three-base mismatching target DNA (tm-DNA): 3'-GGC ATT AAA ATG ATT GGT TCC-5' utilizing IS6110 gene sequences derived from the GenBank database. The single-strand thiolated probe DNA ( $\mathrm{p}$-DNA) was sought. The original genomic sequence was collected from GenBank under accession number AJ242908.1.

Synthesis of laser-scribed graphene nanofiber (LSG-NF). LSG-NF was synthesised on a polyimide (PI) film using a laser-scribing method. The PI film was cleaned using deionised (DI) water and attached to a glass piece for support. A $\mathrm{CO}_{2}$ laser (wavelength $680 \mathrm{~nm}$ ) engraving system (V-460, Universal Laser System, Scottsdale, Arizona, USA) was used to convert the $\mathrm{sp}^{3}$ hybridized atoms of the PI film to $\mathrm{sp}^{2}$ hybridized carbon atoms. The adjustable parameters were the power, speed, and pulse per inch (PPI). The power limits the intensity of the laser that penetrates the PI film, whereas the speed controls the rate at which the nozzle travels in the X-Y direction, and the PPI determines the number of pulses with which the laser beam hits the PI film per inch. The final power, speed, and PPI used to synthesise LSG-NF were $100 \%\left(P_{\max }=30 \mathrm{~W}\right), 30 \%$, and 500 , respectively. The laser-scribing process was conducted in raster mode and performed under ambient conditions.

Green synthesis of silver nanoparticles (AgNPs). Aqueous silver nitrate solution $\left(\mathrm{AgNO}_{3}, 250 \mathrm{~mL}\right.$, $1000 \mathrm{ppm}$ ) was combined with $250 \mathrm{~mL}(1000 \mathrm{ppm})$ of palm oil lignin and mechanically stirred for $2 \mathrm{~h}$ under atmospheric conditions. The mixture was centrifuged at $6000 \mathrm{rpm}$ for $20 \mathrm{~min}$. The residue was extracted and used for development of the biosensor (AgNPs).

Fabrication of DNA bioelectrode. A $4 \times 11 \mathrm{~mm}$ interdigitated electrode (IDE) containing six pairs of electrodes was drawn using CorelDraw software and fabricated by a laser-scribing process. The fabrication process was performed according to the parameters set in the previous step to prepare the laser-scribed graphene nanofibers.

DNA immobilization and hybridization. The IDE was modified by AgNPs to ease covalent bond contact with the thiolated probe DNA. First, $5 \mu \mathrm{L}$ of APTES was dropped on the IDE and incubated for $1 \mathrm{~h}$, followed by $5 \mu \mathrm{L}$ of AgNPs. Thereafter, $5 \mu \mathrm{L}$ of thiolated probe DNA solution was dropped onto the IDE and incubated for $1 \mathrm{~h}$, followed by rinsing with PBS. Target DNA with various concentrations ( $1 \mathrm{fM}$ to $1 \mathrm{nM}$ ) was immobilised on the IDE for hybridisation analysis. In addition, non-complementary and mismatched DNA sequences were thoroughly studied.

Experimental characterization. The surface morphology of the LSG-NF and AgNP-decorated LSGNFs was evaluated by variable pressure (VP) FESEM (Carl Zeiss SUPRA55 VP, Gemini) analysis. A HITACHI HT 7830 high-transmission electron microscope (HRTEM) was used to obtain higher magnification images of LSG-NF and LSG-NF-AgNPs dispersed in ethanol and sonicated for $10 \mathrm{~min}$ before dropping on the copper grid. The structural properties and crystallisation of LSG-NF were studied using Raman spectroscopy (HORIBA Jobin Yvon HR800) and X-ray diffraction (X'Pert ${ }^{3}$ Powder and Empyrean, PANalytical with Cu-Ka radiation $(\lambda=1.54 \AA)$ ). XPS (Thermo Scientific K-Alpha) was used to analyse the immobilisation, hybridisation, and material composition, while FTIR (Perkin Elmer, Spectrum One) was used to analyse the functional groups present after surface modification and functionalization. Electrochemical impedance spectroscopy (EIS) measurements were performed using an Autolab PGSTAT302N by inserting the bioelectrode on an ECOPIA SPCB-01 sample mounting board. The EIS measurements were performed in the frequency range of $100 \mathrm{~Hz}-1 \mathrm{MHz}$ with an $\mathrm{AC}$ amplitude of $0.01 \mathrm{~V}_{\mathrm{RMS}}$, and the results were recorded at room temperature.

\section{Data availability}

Relevant data are included in the "Supporting Information".

Received: 19 November 2020; Accepted: 24 February 2021

Published online: 09 March 2021

\section{References}

1. van Pinxteren, L. A., Ravn, P., Agger, E. M., Pollock, J. \& Andersen, P. Diagnosis of tuberculosis based on the two specific antigens ESAT-6 and CFP10. Clin. Diagn. Lab. Immunol. 7, 155-160 (2000).

2. Li, H. et al. Analysis of the antigenic properties of membrane proteins of Mycobacterium tuberculosis. Sci. Rep. 9, 3042 (2019).

3. Srivastava, S. K., Van Rijn, C. J. M. \& Jongsma, M. A. Biosensor-based detection of tuberculosis. RSC Adv. 6, 17759-17771 (2016).

4. Shojaei, T. R. et al. Development of sandwich-form biosensor to detect Mycobacterium tuberculosis complex in clinical sputum specimens. Braz. J. Infect. Dis. 18, 600-608 (2014). 
5. Nayak, P., Kurra, N., Xia, C. \& Alshareef, H. N. Highly efficient laser scribed graphene electrodes for on-chip electrochemical sensing applications. Adv. Electron. Mater. 2, 1600185 (2016).

6. Griffiths, K. et al. Laser-scribed graphene presents an opportunity to print a new generation of disposable electrochemical sensors. Nanoscale 6, 13613-13622 (2014).

7. Lei, Y., Alshareef, A. H., Zhao, W. \& Inal, S. Laser-scribed graphene electrodes derived from lignin for biochemical sensing. ACS Appl. Nano Mater. 3, 1166-1174 (2020).

8. Lin, J. et al. Laser-induced porous graphene films from commercial polymers. Nat. Commun. 5, 1-8 (2015).

9. Duy, L. X. et al. Laser-induced graphene fibers. Carbon N. Y. 126, 472-479 (2018).

10. Zhang, W., Jia, B. \& Furumai, H. Fabrication of graphene film composite electrochemical biosensor as a pre-screening algal toxin detection tool in the event of water contamination. Sci. Rep. 8, 10686 (2018).

11. Peña-Bahamonde, J., Nguyen, H. N., Fanourakis, S. K. \& Rodrigues, D. F. Recent advances in graphene-based biosensor technology with applications in life sciences. J. Nanobiotechnol. 16, 1-17 (2018).

12. Fenzl, C. et al. Laser-scribed graphene electrodes for aptamer-based biosensing. ACS Sensors 2, 616-620 (2017).

13. Vanegas, D. et al. Laser scribed graphene biosensor for detection of biogenic amines in food samples using locally sourced materials. Biosensors 8, 42 (2018).

14. Su, H. et al. Nanomaterial-based biosensors for biological detections. Adv. Heal. Care Technol. 3, 19-29 (2017).

15. Wang, D., Yan, W., Vijapur, S. H. \& Botte, G. G. Electrochemically reduced graphene oxide-nickel nanocomposites for urea electrolysis. Electrochim. Acta 89, 732-736 (2013).

16. Tan, P., Li, H. S., Wang, J. \& Gopinath, S. C. B. Silver nanoparticle in biosensor and bioimaging: Clinical perspectives. Biotechnol. Appl. Biochem. https://doi.org/10.1002/bab.2045 (2020).

17. Chou, K. S., Lu, Y. C. \& Lee, H. H. Effect of alkaline ion on the mechanism and kinetics of chemical reduction of silver. Mater. Chem. Phys. 94, 429-433 (2005).

18. Shchukin, D. G., Radtchenko, I. L. \& Sukhorukov, G. B. Photoinduced reduction of silver inside microscale polyelectrolyte capsules. ChemPhysChem 4, 1101-1103 (2003).

19. Perumal, V. et al. 'Spotted Nanoflowers': Gold-seeded zinc oxide nanohybrid for selective bio-capture. Sci. Rep. 5, 12231. https:// doi.org/10.1038/srep12231 (2015).

20. Wang, X. J., Wang, W. \& Liu, Y. L. Enhanced acetone sensing performance of Au nanoparticles functionalized flower-like ZnO. Sensors Actuators B Chem. 168, 39-45 (2012).

21. Elumalai, D., Hemavathi, M., Deepaa, C. V. \& Kaleena, P. K. Evaluation of phytosynthesised silver nanoparticles from leaf extracts of Leucas aspera and Hyptis suaveolens and their larvicidal activity against malaria, dengue and filariasis vectors. Parasite Epidemiol. Control 2, 15-26 (2017).

22. Ayad, Z. M., Ibrahim, O. S. \& Omar, L. W. Biosynthesis and characterization of silver nanoparticles by Silybum marianum (silymarin) fruit extract. Adv. Anim. Vet. Sci. 7, 122-130 (2019).

23. Vivek, M., Kumar, P. S., Steffi, S. \& Sudha, S. Biogenic silver nanoparticles by gelidiella acerosa extract and their antifungal effects. Avicenna J. Med. Biotechnol. 3, 143-148 (2011).

24. Goudarzi, A., Lin, L. T. \& Ko, F. K. X-ray diffraction analysis of kraft lignins and lignin-derived carbon nanofibers. J. Nanotechnol. Eng. Med. 5, 1-5 (2014).

25. Ibrahim, M. N. M., Iqbal, A., Shen, C. C., Bhawani, S. A. \& Adam, F. Synthesis of lignin based composites of TiO2 for potential application as radical scavengers in sunscreen formulation. BMC Chem. 13, 1-14 (2019).

26. Liu, L., Qing, M., Wang, Y. \& Chen, S. Defects in graphene: Generation, healing, and their effects on the properties of graphene: A review. J. Mater. Sci. Technol. 31, 599-606 (2015).

27. Dubale, A. A. et al. The synergetic effect of graphene on $\mathrm{Cu} 2 \mathrm{O}$ nanowire arrays as a highly efficient hydrogen evolution photocathode in water splitting. J. Mater. Chem. A 2, 18383-18397 (2014).

28. Bhujel, R. et al. Capacitive and sensing responses of biomass derived silver decorated graphene. Sci. Rep. 9, 1-15 (2019).

29. Ojeda, J. J. \& Dittrich, M. Fourier transform infrared spectroscopy for molecular analysis of microbial cells. In Microbial Systems Biology Vol. 881 (ed. Navid, A.) 187-211 (Humana Press, 2012).

30. Pesala, B. et al. Non-invasive detection of mycobacterium tuberculosis using IR and NIR spectroscopy. in 2012 37th International Conference on Infrared, Millimeter, and Terahertz Waves 1-2 (IEEE, 2012). https://doi.org/10.1109/IRMMW-THz.2012.6380079.

31. Opdahl, A., Petrovykh, D. Y., Kimura-Suda, H., Tarlov, M. J. \& Whitman, L. J. Independent control of grafting density and conformation of single-stranded DNA brushes. Proc. Natl. Acad. Sci. USA 104, 9-14 (2007).

32. Baker, M. J. et al. FTIR-based spectroscopic analysis in the identification of clinically aggressive prostate cancer. Br. J. Cancer $\mathbf{9 9}$, 1859-1866 (2008).

33. Wang, L. et al. Development of an electrochemical DNA biosensor with the DNA immobilization based on in situ generation of dithiocarbamate ligands. Bioelectrochemistry 88, 30-35 (2012).

34. Ramulu, T. S. et al. Nanowires array modified electrode for enhanced electrochemical detection of nucleic acid. Biosens. Bioelectron. 40, 258-264 (2013).

35. Perumal, V. et al. Gold nanorod embedded novel 3D graphene nanocomposite for selective bio-capture in rapid detection of Mycobacterium tuberculosis. Biosens. Bioelectron. 116, 116-122 (2018).

36. Gopinath, S. C. B. et al. Aptamer-based determination of ATP by using a functionalized impedimetric nanosensor and mediation by a triangular junction transducer. Microchim. Acta 184, 4425-4431 (2017).

\section{Acknowledgements}

V.P. thanks Universiti Teknologi PETRONAS (UTP) for support with the experiments through URIF Grant 015LBO-021 and for providing opportunities to conduct the research in the Centre of Innovative Nanomaterials and Nanodevices. The authors also thank Indra Gandi Subramani and Nur Syahirah Husna bte Suhaimi for their technical support. Appreciation also goes to all the team members and staff at the School of Chemical Sciences, Universiti Sains Malaysia (USM), the Centre of Innovative Nanomaterials and Nanodevices (COINN), UTP.

\section{Author contributions}

V.P. and M.J.Y.T. conceived and developed the LSG-NF. I.N.J. and N.S.H.S. developed AgNPs. M.J.Y.T. carried out the experiments and drafted the manuscript. S.C.B.G., M.N.M.I., P.B.R., and W.W.L. proofread the manuscript. V.P. and P.B.R. supervised the work. All authors analysed the results and contributed to the discussion presented in the manuscript.

\section{Competing interests}

The authors declare no competing interests. 


\section{Additional information}

Supplementary Information The online version contains supplementary material available at https://doi. org/10.1038/s41598-021-85039-2.

Correspondence and requests for materials should be addressed to V.P. or S.C.B.G.

Reprints and permissions information is available at www.nature.com/reprints.

Publisher's note Springer Nature remains neutral with regard to jurisdictional claims in published maps and institutional affiliations.

(c) (i) Open Access This article is licensed under a Creative Commons Attribution 4.0 International License, which permits use, sharing, adaptation, distribution and reproduction in any medium or format, as long as you give appropriate credit to the original author(s) and the source, provide a link to the Creative Commons licence, and indicate if changes were made. The images or other third party material in this article are included in the article's Creative Commons licence, unless indicated otherwise in a credit line to the material. If material is not included in the article's Creative Commons licence and your intended use is not permitted by statutory regulation or exceeds the permitted use, you will need to obtain permission directly from the copyright holder. To view a copy of this licence, visit http://creativecommons.org/licenses/by/4.0/.

(C) The Author(s) 2021 